\title{
SMART SECURITY BASED ON AUTOMATIC NUMBER PLATE RECOGINITION
}

\author{
Mr. R. Navin kumar, M C A, M Phil*, Mr. S. K. Sedhu Raman, M C A ** \\ *(Assistant Professor, Department of Computer Applications, \\ Nandha Engineering College (Autonomous), \\ Erode, Tamil Nadu, India \\ Email: navinsoccer07@gmail.com) \\ ** (Final MCA, Department of Computer Applications, \\ Nandha Engineering College (Autonomous), \\ Erode, Tamil Nadu, India \\ Email: sedhuramanrko@gmail.com)
}

$* * * * * * * * * * * * * * * * * * * * * * * *$

International Journal of Computer Techniques - Volume 7 Issue 2, March 2020

\section{Abstract:}

This venture is entitled as "SMART SECURITYBASED ON AUTOMATIC NUMBER PLATE RECOGNITION", is developed via the usage of MATLAB as the front end. This automation can predict the quantity plate from an enter photo and compares the anticipated number plate with registered automobiles quantity plate then it's going to displays the end result to the user. Automatic Number Plate Recognition is an picture processing technology and important subject of studies that identifies automobiles via their wide variety plates in the course of which the amount plate facts is extracted from vehicle photo or from series of photographs without direct human intervention. ANPR consist of four phases: Preprocessing, number plate extraction, character segmentation, characters recognition. This paper gives an efficient approach for number plate extraction from pre-processed vehicle's enter photograph using morphological operations, edge detection and connected factor the enter image is first pre-process the usage of iterative bilateral clear out and adaptive histogram equalization. The important goal of this assignment is to examine the predicated range plate from an input picture to registered vehicle photos. If the range plate from input photo fits the registered vehicle's wide variety plate it suggests the precise end result for user. Here the guide attempt for comparing variety plates with registered number plate has ignored.

Keywords - ANPR, GIS, GPS, NP, NN.

$* * * * * * * * * * * * * * * * * * * * * * * *$

\section{INTRODUCTION}

Automatic variety plate popularity technique can be applicable for modern society. This method may be used to display the vehicles while it's far passing through greater touchy areas. Through this application it could restriction the blocked vehicles and experience the Recognition device is an important area of pc science involved with spotting
It is critical optical characters popularity, voice recognition and handwriting popularity. It makes use of techniques from statistics, gadget getting to know and different area. Typical applications are textual content class to renowned unique kind of texts like unsolicited mail and non junk mail Emails, speech recantation for specified functions like translating extraordinary languages to English, hand written recognition for postal codes, or the 
automatic face popularity which deals with digital pix as input to the pattern reputation In preceding years, several kinds of photograph analysis strategies are applied to research the agricultural pictures like culmination and vegetables, for popularity and category In conventional image search systems, user commonly find it difficult to define or describe their in search of demands with recognize to photographs even when popular search hobbies appear, attributable to an absence of unique information of explicit intent with the aid of forming seek queries. So that it requires new proposed system to overcome those troubles observed in existing system. Spaces between characters are also measured in order that words may be extracted out. In/additionolqbaracter\&/6qncben of any colors inside the give photograph. It compares the anticipated number plate from an input image to registered vehicle's wide variety plate and suggests the end result to the users.

With the growing quantity of vehicles, finding a parking lot can be a serious trouble today for an outsized number of students and faculty at Educational Institutions. Most of the automobile parks are managed manually by security guards who don't maintain a track of the quantity of motors entering and exiting the premises. Hence, the vehicle driver need to keep circling the parking lot with a view to are seeking for out a vacant slot resulting in a wastage of your time, to no longer mention the anxiety and frustration of The absence of the safety guards may additionally also result in automobile thefts.

\section{LITERATURE REVIEW}

Rakesh Agrawal Jerry Kiernan et al [2004] [1] describe the piracy of digital assets including software program, images, video, audio and text has lengthy been a subject for owners of those Protection of these assets is generally based upon the insertion of virtual watermarks into the statistics The watermarking software introduces small errors into the item being watermarked. These intentional errors are referred to as marks and all the marks collectively constitute the watermark. The marks must not have a substantial effect on the usefulness of the information and they need to be positioned in such a way that a malicious user can't break them with out making the statistics much much less Thus, watermarking does no longer prevent copying, but it deters unlawful copying via supplying a way for setting up the unique ownership of a redistributed copy.

These versions include:

- A multimedia item includes a massive quantity of bits, with enormous redundancy. Thus, the watermark has a large cover in which to hide. A database relation includes tuples, every of which represents a separate item. The watermark desires to be unfold over those separate items.

- The relative spatial/temporal positioning of numerous quantities of a multimedia item normally

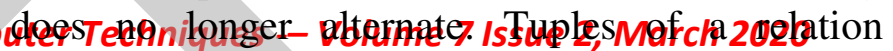
however represent a set and there may be no implied ordering among them.

- Portions of a multimedia item can not be dropped or replaced arbitrarily without causing perceptual adjustments within the object. However, the pirate of a relation can in truth drop a few tuples or opportunity them with tuples from different individuals of the family.

Claudio Lucchese et al [2001] [2] describe the sharing is an crucial factor of scientific or agency collaboration. However, information proprietors are also undertaking with the safety of their rights on the datasets, it is many instances have been acquired after expensive and laborious procedures. The ease of facts alternate via the Internet has compounded the want to bring together technological mechanisms for efficaciously protecting one's intellectual or pragmatic belongings. Trajectories abound in applications which incorporates GPS tracking experiments, video and movement capture statistics, and even photo shapes can be considered as 2-dimensional We offer possession assurances on such datasets using watermarking principles. While there is a rich literature on watermarking for multimedia datasets, previous art work is primarily involved with watermarking a single object and not a group of Here, we take into account the watermarking trouble from a state-of-the-art perspective, through focusing at the additional preservation of the interrelationship between 
The method embeds a mystery key in each of the dataset devices, distorting them imperceptibly, at the same time as taking particular attention in retaining the particular neighbouring item. We name this operation Neighbour Preserving (NP) watermarking. Guaranteeing safety of the nearest devices is very important for an array of are looking for and mining operations, including similarity seek or Nearest-Neighbor(NN)-classification. Contrary to privacy-maintaining tactics for statistics-mining that first upload noise and then reconstruct the particular facts distributions based totally at the diagnosed noise model, our technique learns/calculates the most important quantity of noise that can be added, so that nearest neighbors

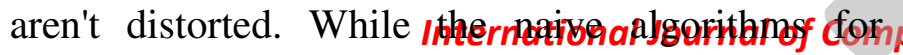
figuring out the said watermarking strength are costly, we show efficient strategies of speeding up the way making it more than 2 orders of significance faster, thus allowing the technique to be applicable to huge datasets.

Victor R. Doncel, Nikos et al [2000] [3] describe a watermark is a hidden information interior a virtual signal, used mostly for copyright protection of multimedia information. Its main capabilities are the imperceptibility of the imposed medicinal capsules and its persistence in opposition to processing (attacks) that may result in its removal, either intentionally or unintentionally. A stylish framework for virtual water marking has been presented, whereas gives an extremely good examine of the watermarking principles and techniques. Digital watermarking has been specifically applied to nonetheless photograph, audio and video statistics. However, little work has been completed in watermarking vector graphics information, that are commonly utilized in Geographic Information Systems (GIS) or in Computer Aided Design

Digital watermarking is a polygonal line, which can be a key photos primitive in vector photographs data and consequently may be used for the copyright protection of such information. Furthermore, the technique can be used for the watermarking of MPEG-4 natural video facts by using watermarking the outline of the Video Objects in MPEG-4 stream. In that case, the approach need to be followed with the resource of a manner of extrapolating current textures in case the watermarked boundary defines a bigger place than the authentic This paper extends the artwork presented. The identical embedding method is observed here, and efforts attention the format of a trendy, improved overall performance detector. Theoretical and experimental analysis display that a huge development in detection overall performance can be finished if the facts of the watermarked polygon are taken into

Rakesh Agrawal and Jerry Kiernan et al [2006][9] describe watermarking database family individuals to discourage their piracy, pick out the precise trends of relational information which pose new demanding conditions for watermarking, and

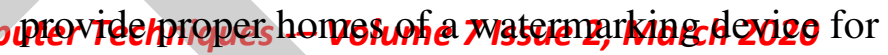
relational A watermark can be applied to any database relation having attributes which might be such that changes in some of their values do now not have an impact at the applications. An effective watermarking approach geared for relational facts. This method guarantees that a few bit positions of a number of the attributes of some of the tuples contain specific values. The tuples, attributes inside a tuples, bit positions in an attribute, and unique bit values are all algorithmically determined below the manipulate of a non-public key acknowledged only to the owner of the data

N. F. Johnson, Z. Duric [2000][10] describe the piracy of virtual property such as software program application, images, video, audio and textual content has prolonged been a hassle for owners of Protection of those assets is generally based sincerely upon the insertion of digital watermarks into the records. The watermarking software introduces small mistakes into the object being watermarked. These intentional mistakes are called marks and all of the marks together represent the watermark. The marks must now not have a massive impact on the usefulness of the statistics and they need to be located in such a way that a malicious purchaser can not spoil them without making the data an awful lot a good deal less beneficial. Thus, watermarking does no longer prevent copying, however it deters illegal copying by way of imparting a method for installing the original possession of a redistributed copy. The developing use of databases in applications beyond 
"behind-the-firewalls records processing" is growing a similar want for watermarking databases.

\section{RELATED WORK}

First of all the schooling set is being uploaded in a undertaking for finding the numbers, alpha and unique characters. A few studies attempts deal with the information-in search of problem in terms of improving the surfing experience. Several other research efforts focus on typical key-wordprimarily based search scenarios, primarily tackling the challenges of question understanding issues in a search system, consisting of query classification, question disambiguation, and question A quantity of works leverage contextuahaquensfor ufacilitatigg search motive expression and query augmentation. In the last few years, exploratory seek has been regarded by way of many in the studies community as an opportunity for enhancing the standard seek. Exploratory seek is described as a specialization of information-seeking behavior, which is commonly depicted via three varieties of activities lookup, learn investigate, mainly pertinent to the learn and check out sports. As the web has become the first choice for information seekers, people assume it to serve other forms of statistics needs and search engines need to strive to provide services beyond Consequently, research has targeted on defining a broader set of information behaviors to find out about the conditions when a user is, or feels, limited by way of simplest having the potential to carry out a key-word seek, especially while visual statistics is involved.

\section{MODULES}

\section{A. Training set - alphabet image addition}

The upload alphabet form is used to feature the alphabet person records within the application details along with individual from the combo box control, photo report direction from the open document dialog manage and photograph inside the image box manage and shop these records inside the "images" table.

\section{B. Training set - number image addition}

The upload quantity form is used to add the range records in the application info such as person from the combination box manage, photo record course from the open file dialog manage and photo inside the picture box manipulate and store these statistics in the "images" table.

\section{Training set - special character image addition}

The add special man or woman shape is used to add the unique person facts within the application details consisting of unique man or woman from the combo box control, photo record path from the open document dialog control and picture inside the photo box manipulate and save these information within the "images"

\section{puter Techniques --Volume 7 Issue 2, March 2020}

\section{Select image}

In this module, the picture document is selected. Using open document dialog manipulate, the image record is selected. The picture type can be any format (e.G., bmp, jpg, gif, tiff, etc). Both grey scale and coloration picture may be selected.

\section{E. Scene text recognition}

In this module, the selected image file is taken for processing. Then threshold value is about so that one-of-a-kind size of man or woman aside from in training set also can be extracted from the given photo. Spaces among characters also are measured in order that words may be extracted out. In addition, characters can be of any colors within the give image. Using Character Skeleton and Character Boundary extraction, the individual is recognized.

\section{EXISTING WORKS}

Firstly, given a synthesized character patch from the training set, the existing system obtains character boundary and character skeleton by applying discrete contour evolution and skeleton pruning on the basis of evolution. The evolution recognize characters and shows to the user, and an edge detection is not plays a important role in it. Then it is not compares the predicted number from the given input image to registered vehicles number plate. 


\section{Drawbacks}

- Only character level recognition is considered.

- Edge detection is not used.

- Shows only the text in the number plate.

- Does not compare the predicted number plate to registered vehicle's number plate.

- All type of image format is not applicable

\section{PROPOSED SYSTEM}

All the existing system approach is carried out in proposed system. In addition, any image type can be given as source image. Spaces between characters are also measured so that words can be extracted out. In additiohntekaptieers eaprbel of GAy colors in the give image. It compares the predicted number plate from an input image to registered vehicle's number plate and shows the result to the users.

\section{Advantages}

- Any Image type can be taken for text extraction.

- Edge detection plays an important role in it.

- Shows the number plate and compares with registered vehicle's number plate.

- All type of image format is applicable.

\section{EXPERIMENTAL RESULT}

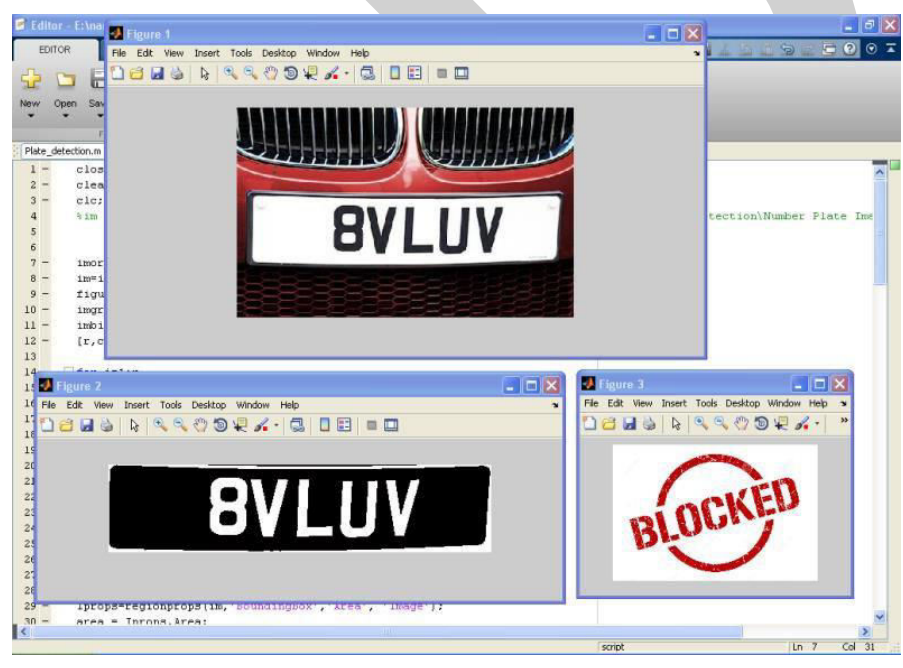

Figure(1)

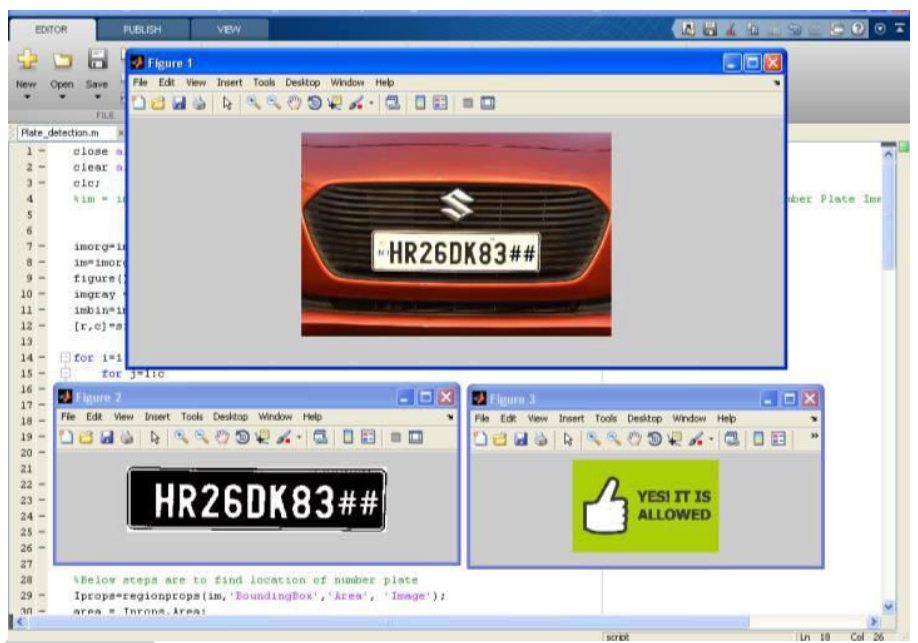

Figure(2)

uter Techniques - Volume 7 Issue 2, March 2020

Figure(1) shows the given input image to the user. Then the user can see the predicted number plate from an input image. After the number plate recognition it will compares he predicted number plate to registered vehicle's number plate. From figure (2) the user can found the result weather it is registered or not.

\section{CONCLUSION}

The new device gets rid of the difficulties within the current device. It is evolved using Matlab as front stop for an powerful photograph processing. The machine can be very rapid and any image format can be considered or retaken at any level. This research artwork can be very precise in reducing the paintings and attaining the object. It will reduce plate popularity time than existing gadget. The purchaser can with out trouble understand the from output image. This art work will beneficial resource for the future development.

- Data is entered in all photograph formats.

- The related photographs can be searched with more input.

- Provides correct result.

The studies paintings has covered almost all the requirement. Further necessities and enhancements can with out troubles be done due to the fact the coding in specifically mounted or modular in nature. 
Improvements can be appended by changing the triumphing modules or which embody new modules. Several areas to be advanced in destiny, so the application ought to be upgraded for the present day ones required and it's far viable to adjustments steady with new necessities and specifications.

\section{REFERENCES}

[1] Rakesh Agrawal Jerry Kiernan et al , "Skeleton pruning by contour partitioning with discrete curve evolution," IEEE Trans. Pattern Anal. Mach. Intell., vol. 29, no. 3, pp. 449-462, Mar. 2004.

[2] Claudio Lucchese et al, "A weighted finite-state framework for correcting errors in natural scene OCR," in Proc. 9th Int. Conf. Document Anal. Recognit., Sep. 2001, pp. 889-893.

[3] Victor R. Doncel, Nikos et al, "Automatic detection and recogniton of

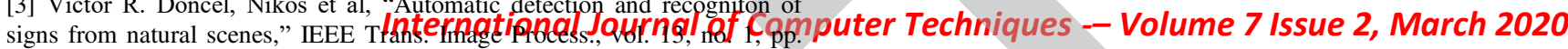
87-99, Jan. 2000.

[4] A. Coates et al., "Text detection and character recognition in scene images with unsupervised feature learning," in Proc. ICDAR, Sep. 2011, pp. 440-445.

[5] N. Dalal and B. Triggs, "Histograms of oriented gradients for human detection," in Proc. IEEE Conf. Comput. Vis. Pattern Recognit., Jun. 2005, pp. 886-893.

[6] T. de Campos, B. Babu, and M. Varma, "Character recognition in natural images," in Proc. VISAPP, 2009.

[7] B. Epshtein, E. Ofek, and Y. Wexler, "Detecting text in natural scenes with stroke width transform," in Proc. CVPR, Jun. 2010, pp. 2963-2970.

[8] P. F. Felzenszwalb, R. B. Girshick, D. McAllester, and D. Ramanan, "Object detection with discriminatively trained part-based models," IEEE Trans. Pattern Anal. Mach. Intell., vol. 32, no. 9, pp. 1627-1645, Sep. 2010.

[9] Rakesh Agrawal and Jerry Kiernan et al, "Learning shape prior models for object matching," in Proc. CVPR, Jun. 2006, pp. 848-855.

[10] N. F. Johnson, Z. Duric, "Text extraction and document image segmentation using matched wavelets and MRF model," IEEE Trans. Image Process., vol. 16, no. 8, pp. 2117-2128, Aug. 2000. 